\title{
Remote Monitoring of Patient-Reported Outcomes in Ulcerative Colitis: A Prospective Real-World Pilot Study
}

\author{
Shaji Sebastian ${ }^{1} \cdot$ Jenna Roberts ${ }^{2} \cdot$ John Waller $^{2} \cdot$ Davneet Judge $^{2} \oplus \cdot$ Chloe Brown $^{4} \cdot$ Ruth Davies $^{4}$. \\ Sumesh Kachroo ${ }^{3}$
}

Published online: 21 March 2019

(c) The Author(s) 2019

\begin{abstract}
Introduction The 6-point version of the Mayo score relies on two patient-reported outcomes (PRO2): stool frequency and rectal bleeding. We assessed the feasibility and acceptability of remote online PRO2 reporting for golimumab-treated ulcerative colitis (UC) patients.

Patients and Methods This was a UK-based, multi-centre, prospective, real-world, non-interventional pilot study. Eligible patients completed PRO2 scores at baseline and every 4 weeks over a period of 6 months. Demographics were collected at baseline and a satisfaction questionnaire was completed at study end. Each patient provided data anonymously via an online platform. Results Fifty-two patients enrolled in the study. Mean (SD) patient age was 40.8 (13.6); 52\% were male. Patients provided data on a personal computer (44\%), mobile phone (38\%) or tablet (18\%). Forty-seven (90\%) patients completed the baseline questionnaire within the accepted time range. Subsequent scores were reported on time by eligible patients with a success rate of $94 \%, 92 \%, 90 \%, 87 \%, 90 \%$ and $81 \%$ at end of months $1-6$, respectively.

Conclusions Remote monitoring of PRO2 in UC was feasible amongst the sample tested. Of those initially willing to provide data in this way, attrition was low. Formal roll-out of this system could be used to support a more frequent assessment of UC symptoms without over-burdening the healthcare system.
\end{abstract}

\section{Key Points for Decision Makers}

This pilot study assessed the feasibility of measuring disease activity via the 6-point version of the Mayo score (PRO2 score) in ulcerative colitis patients.

The results from the study support the feasibility of using remote monitoring systems (i.e. mobile phones, tablets or personal computers) to capture PRO2 scores.

Patients within the study reported high satisfaction and acceptance of remote monitoring, whilst also stating they would consider this method of data reporting in the future.

Electronic supplementary material The online version of this article (https://doi.org/10.1007/s41669-019-0121-8) contains supplementary material, which is available to authorized users.

Davneet Judge

Davneet.Judge@adelphigroup.com

IBD Unit, Hull and East Yorkshire Hospital NHS Trust, Hull, UK

Adelphi Real World, Bollington, UK

Merck \& Co., Inc, Kenilworth, NJ, USA

4 Merck Sharpe \& Dohme Ltd, Hertfordshire, UK

\section{Introduction}

Ulcerative colitis (UC) is a chronic inflammatory disease of the colon characterised primarily by bloody diarrhoea and inflammation of the mucosa of the colon and rectum [1]. Treatment goals include inducing and maintaining remission, although mucosal healing may also be an important aim [2]. The introduction of biologic therapies has provided new opportunities for improved disease control, including mucosal healing. Several options are now available, including infliximab, adalimumab, golimumab, tofacitinib and vedolizumab.

Golimumab is a subcutaneously administered, fully human anti-tumour necrosis factor (TNF) antibody indicated for moderate-to-severe active UC. In the PURSUIT (Program of Ulcerative Colitis Research Studies Utilizing an Investigational Treatment) phase III clinical programme, clinical remission and clinical response were assessed with Mayo scores, which are a composite measure of disease activity comprising stool frequency, rectal bleeding, endoscopic findings and a global physician assessment. The clinical trial data indicated that significantly more patients on golimumab maintenance therapy, 
relative to placebo, maintained clinical response at week 54, and achieve remission at week 30 and week 54 [3].

In routine clinical practice, regular assessments of disease activity can help ensure that treatment goals are met, the patient is receiving the most appropriate therapy, and the risk of complications is monitored. Endoscopic evaluation has been described in the literature as the gold standard for disease monitoring in UC $[4,5]$. However, its invasive nature and the associated cost and patient burden limit the frequency that endoscopy can practically be performed $[5,6]$.

Clinical symptoms are important in UC and may include abdominal pain, fever, diarrhoea, increased stool frequency and rectal bleeding. The latter two symptoms are selfreported by patients as part of a 6-point version (PRO2) of the Mayo score disease activity index [7]. Measuring $\mathrm{PRO} 2$ scores is non-invasive, is not reliant upon laboratory tests or face-to-face involvement of a clinician, and correlates well with other disease severity measures [8-10]. This brief instrument could provide a useful and convenient way of monitoring disease activity, including remotely, for example, via regular patient reports of these outcomes on a smartphone, tablet or computer. Such reporting of PRO2 scores may allow physicians to better monitor the pattern of disease activity over time, without overburdening the patient or the healthcare system. Indeed, greater use of information communication technology (ICT), including mobile devices in healthcare (eHealth), has been identified as a priority by the European Commission [11].

In line with this, remote monitoring systems (e.g. telephones, smartphones and web portals) for managing chronic diseases are currently being explored across a broad range of therapy areas, including UC [11, 12]. Initial data from a small qualitative study $(n=24)$ suggests that inflammatory bowel disease patients are very receptive to this idea [13,14].

The primary objective of the present pilot study was to assess the feasibility and acceptability of remote online reporting of disease activity using the PRO2 scores for golimumab-treated UC patients. Scores were collected every 4 weeks over a 6-month period and patients also completed a satisfaction questionnaire at study end.

\section{Patients and Methods}

\subsection{Study Design and Patient Population}

This was a UK-based, multi-centre, non-interventional, prospective, real-world pilot study of golimumab-treated UC patients involving a brief Case Report Form (CRF) and online data collection of patient-reported outcomes (REC approval number: 16/LO/1880).
Patients were eligible to participate in this study if, at the time of enrolment, they were $\geq 18$ years old, had a diagnosis of UC, had been treated with golimumab for at least 4 weeks prior to enrolment, volunteered to participate in the study for a period of 6 months (although they had the right to withdraw at any point), were not involved in an interventional clinical trial for UC, were being treated as an outpatient at the time of enrolment and provided written informed consent. Eligible patients who consulted with their UC healthcare team during the recruitment phase were invited to participate in the study on a consecutive basis and patients were recruited from ten secondary care sites across the UK. All patient-reported outcome measures were provided in an online format; all patients therefore required access to a home computer, tablet or smartphone. Device choice was driven by patient preference and/or accessibility. All treatment decisions for participating patients were made outside of the research and were not influenced by the patient's involvement in the study. Patients were informed at the start of the study that their responses would not be shared with the healthcare team, and, therefore, their participation within the study would have no impact on the care that they received. Instead, the data were transferred directly to an independent research team. No patient identifiable information was collected throughout the study and all responses were anonymised.

\subsection{Study Measures}

\subsubsection{Patient-Reported Data}

Patient-reported PRO2 scores were captured online at baseline (defined as the date of patient enrolment into the study) and then every 4 weeks over a period of 6 months (up to 7 scores per patient at the end of the study). The $\mathrm{PRO} 2$ questionnaire consists of two separate questions (stool frequency and rectal bleeding), with four response options each, scored from normal (no disease symptoms) to most severe symptoms (0-3) (further detail can be found in "Appendix"). A $\mathrm{PRO} 2$ score of 0 indicates inactive UC and a score of 6 indicates active disease and spontaneous bleeding. Patients were also asked to complete additional validated patientreported outcome (PRO) measures on health-related quality of life and work productivity at baseline and study end but, as these data are not pertinent to the primary objective, they are not presented here.

Additional baseline questions in the patient questionnaire included patient demographics and recent disease severity. The severity question asked patients to rate how severe their symptoms had been over the 1 month prior to enrolment on a 5-point scale from 'not severe at all' to 'worst imaginable'. Disease severity was also assessed at study end using the same scale; here patients rated their symptoms over the 
previous 6 months, i.e. the study period where PRO2 scores were captured.

An evaluation/satisfaction questionnaire was also included at study end to assess patient acceptance of remote reporting and overall satisfaction with providing the PRO2 scores electronically every 4 weeks (questionnaire provided in electronic supplementary material). Seven of these ten questions were rated on a 5-point scale, where 0 corresponded to 'not at all' and 4 corresponded to 'very much so'. Patients were asked how often they could realistically use online reporting in future with five options ranging from less than once a month to up to twice per week. An overall satisfaction question was also included with three response options (not at all satisfied, neither satisfied nor dissatisfied, and very satisfied). Finally, patients were asked if they would like to use online reporting in future for communication with their healthcare team (yes, no or maybe).

All questionnaires were administered via a web-based application (Open Clinica), and a link to this site was sent to patients via email and/or text. The link directed patients to the relevant questionnaire measures, which could be completed on their choice of device. For the baseline questionnaires only, patients indicated whether they had used a personal computer, smart phone or tablet to provide the requested data. Once patients had submitted their questionnaire data, the link for that data collection timepoint was closed and patients could not view or modify their responses. Notifications and reminders were utilised as part of the study, in order to enhance response rates; two notifications were sent out prior to each data capture time point (one the day before and one on the actual day). If no PRO2 score was provided by the patient at the designated time point, up to three standardised text/email reminders were sent out on consecutive days. If the PRO2 response was not completed by the third reminder, no further reminders were issued and the link to the questionnaire closed until the next score was due the following month.

\subsubsection{Physician-Reported Data}

Physicians or delegated site personnel completed two online CRFs for each recruited patient capturing retrospective secondary data from medical records. The baseline CRFs captured key demographic, clinical, and treatment information at baseline and basic healthcare resource use (e.g. number of appointments) for up to 6 months prior to enrolment. At the end of the 6-month follow-up period, an additional section of the CRF became available to capture retrospective data from the study period (i.e. any changes to treatment occurring during the study period such as golimumab discontinuation or switch to another therapy).
Data were collected from March 2017 to March 2018.

\subsection{Statistical Analyses}

Descriptive statistics are shown for all data using Version 15.0 of the Stata software package (StataCorp LLC, College Station, TX, USA) and SPSS Data Collection Survey Reporter 7.

To determine acceptance of the PRO2 remote reporting requirements, patients were classified as 'successfully completed' if each PRO2 score was provided within 3 days of the scheduled time point. Results describe the number of patients failing to report scores at each time point. Data from the satisfaction/evaluation questionnaire are summarised with descriptive statistics.

\section{Results}

\subsection{Patient Characteristics}

A total of 52 patients enrolled in the study and 27 (52\%) were male. Mean (SD) age was 40.8 (13.60) years. Patients were diagnosed with UC for a mean (SD) of 9.0 (7.78) years and had been receiving golimumab for a mean (SD) of 11.6 (9.50) months at the time of study enrolment. Most patients (41 patients, 79\%) were biologic-naïve prior to golimumab treatment. By the end of the study, four (8\%) patients had discontinued golimumab treatment (Table 1). Patients selfreported symptom severity at both baseline (severity experienced 1 month before enrolment) and at the study endpoint (experienced over the 6-month study duration). The proportion of patients experiencing very severe and somewhat severe symptoms was similar at baseline $(n=12,24 \%)$ and 6 months $(n=11,24 \%)$ (Table 2).

\subsection{Patient-Reported Data Completion and Dropout Rates}

The majority of patients enrolled in the study completed all questionnaires over the 6-month study period $(n=46$, $89 \%$ ) (Table 3). In total, 6 (11\%) patients failed to provide a score at various points in the study. Most patients who provided the required data did so within the allotted 3-day time period. The highest proportion of late responses were recorded at the final study time point where 4 of the 46 patients who were still in the study responded to their final questionnaire late (Table 3). Fifty (96\%) patients completed the baseline PRO2 measure and subsequent scores were successfully completed by patients at a rate of 47 (90\%), 49 (94\%), 48 (92\%), 47 (90\%), 45 (87\%), 47 (90\%), 
Table 1 Patient demographic data collected from medical charts

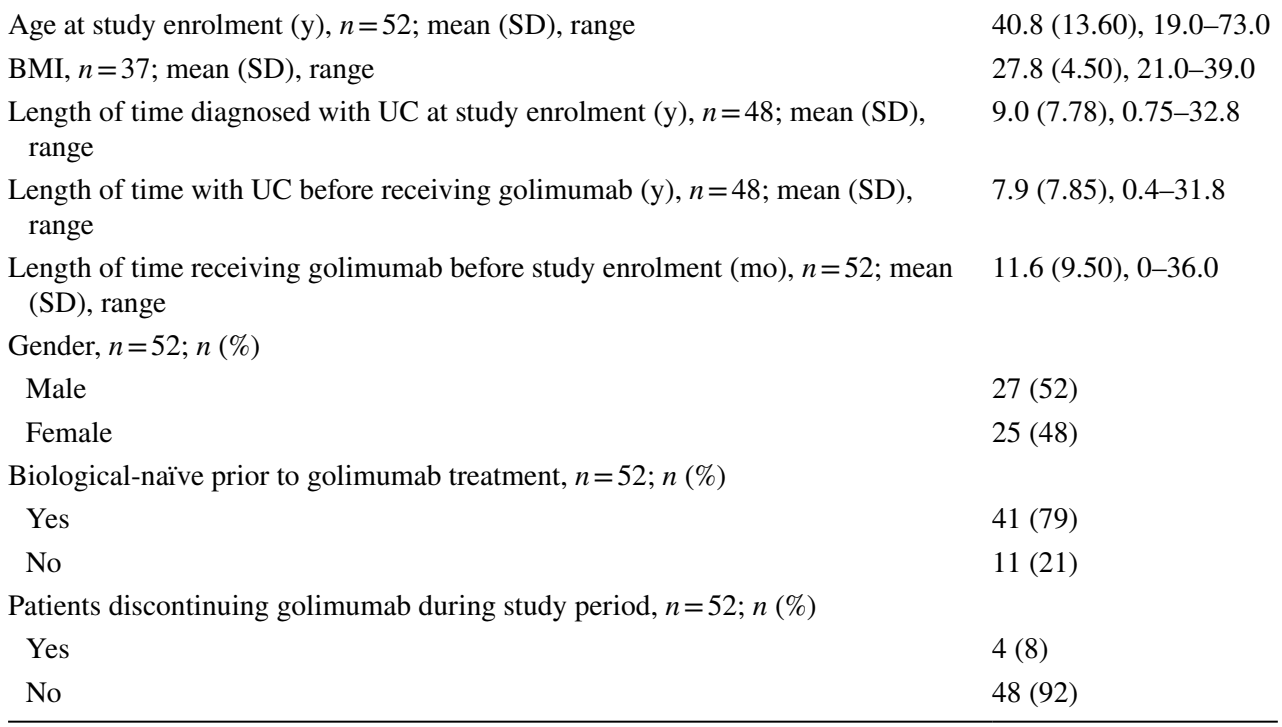

mo months, $S D$ standard deviation, $U C$ ulcerative colitis, $y$ years
Table 2 Patient-reported symptom severity

\begin{tabular}{lll}
\hline Symptom severity, $n(\%)$ & $\begin{array}{l}\text { Baseline } \\
n=50\end{array}$ & $\begin{array}{l}\text { Over 6 months } \\
n=46\end{array}$ \\
\hline Not severe at all & $19(38)$ & $14(30)$ \\
Mild & $19(38)$ & $21(46)$ \\
Somewhat severe & $9(18)$ & $8(17)$ \\
Very severe & $3(6)$ & $3(7)$ \\
Worst imaginable & 0 & 0 \\
\hline
\end{tabular}

and $42(81 \%)$ at baseline and the end of months 1 to 6 , respectively (Table 3). As assessed at baseline, 22 patients (44\%) provided data on a personal computer, 19 (38\%) on a smartphone, and $9(18 \%)$ on a tablet. Device choice was driven by patient preference and did not necessarily remain stable throughout the study.

\subsection{Patient-Reported Perceptions on the Acceptability of Remote Data Reporting}

Patient-reported satisfaction with remote monitoring was high, with $35(76 \%)$ of patients stating they were very satisfied with online reporting throughout the study and 33 (72\%) stating that they would like to use online reporting in the future for communicating with their doctor or nurse (Table 4). The majority of patients stated they would realistically use remote reporting at least once a month $(51,99 \%)$ (Table 4). Patients also reported that remote reporting was not complicated to use at all $(42,91 \%)$, felt it did not take up
Table 3 Patient-reported data completion rates and drop-out rates

\begin{tabular}{|c|c|c|c|c|c|c|c|}
\hline & $\begin{array}{l}\text { Baseline } \\
\text { question- } \\
\text { naire }\end{array}$ & Month 1 & Month 2 & Month 3 & Month 4 & Month 5 & $\begin{array}{l}\text { 6-month } \\
\text { question- } \\
\text { naire }\end{array}$ \\
\hline \multicolumn{8}{|c|}{ Rates of questionnaire and PRO2 completion and drop-out rates, $n=52$} \\
\hline Completed & $50(96)$ & $49(94)$ & $49(94)$ & $47(90)$ & $47(90)$ & $47(90)$ & $46(89)$ \\
\hline Successfully completed ${ }^{\mathrm{a}}$ & $47(90)$ & $49(94)$ & $48(92)$ & $47(90)$ & $45(87)$ & $47(90)$ & $42(81)$ \\
\hline Late but complete & $3(6)$ & 0 & $1(2)$ & 0 & $2(4)$ & 0 & $4(8)$ \\
\hline Not completed & $2(4)$ & $3(6)$ & $3(6)$ & $5(10)$ & $5(10)$ & $5(10)$ & $6(11)$ \\
\hline \multicolumn{8}{|c|}{ Mode of questionnaire completion, $n=50$} \\
\hline Personal computer & $22(44)$ & & & & & & \\
\hline Smartphone & $19(38)$ & & & & & & \\
\hline Tablet & $9(18)$ & & & & & & \\
\hline
\end{tabular}

${ }^{\mathrm{a}}$ Indicates that data was provided within three-days of the scheduled time point

All data are shown as $n(\%)$ unless otherwise indicated 
much of their time at all $(41,89 \%)$ and that it did not interfere with their usual activities at all $(46,100 \%)$ (Table 4).

Again, the majority of patients felt that online reporting could very much be important for reporting their health information for their doctor or nurse $(31,67 \%)$ and that it would also very much have a positive impact on their health (26, 57\%) (Table 4). Full results of the patient satisfaction and acceptance survey of reporting report is presented in Table 4.

\subsection{Disease Activity}

Figure 1 illustrates the PRO2 scores collected over the 6-month study duration. At baseline, patients reported a mean (SD) PRO2 score of 0.9 (1.27). Overall, an increase in disease activity was observed during the study. The mean (SD) PRO2 score at the study endpoint was 1.4 (1.50). Patients who reported experiencing more severe symptoms during the study also had higher PRO2 scores relative to patients who reported experiencing none to mild severity symptoms (Fig. 1).

\subsection{Healthcare Resource Utilisation}

A low number of routine consultations, telephone clinics and hospitalisations were reported for patients at both

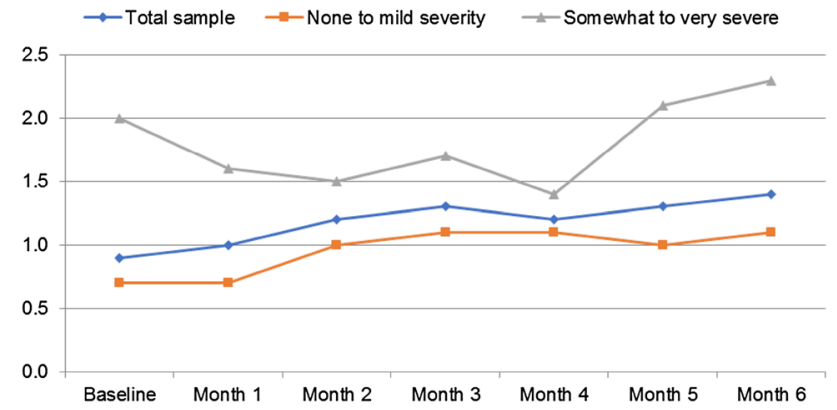

Fig. 1 Patient-reported PRO2 scores collected over the 6-month study duration. Self-reported symptom severity experienced over the duration of the study (data captured retrospectively at 6-month study endpoint) has been used to stratify the data (total $n=50$; none to mild severity $n=35$; somewhat to very severe $n=11$ ). Note, four patients were lost to follow-up at the 6-month time point

baseline (defined as 6 months preceding study enrolment) and 6-month study endpoint (Table 5). The mean number of routine consultations (mean [SD], baseline 2.1 [1.51]; 6 months 1.7 [1.86]), telephone clinics (baseline 0.8 [1.23]; 6 months 0.3 [0.56]) and number of hospitalisations (outside of routine consultations) (baseline $<0.1$ [0.19]; 6 months 0.2 [0.69]) reported remain similar at both time points.

Table 4 Patient-reported acceptance and satisfaction with remote reporting $(n=46)$

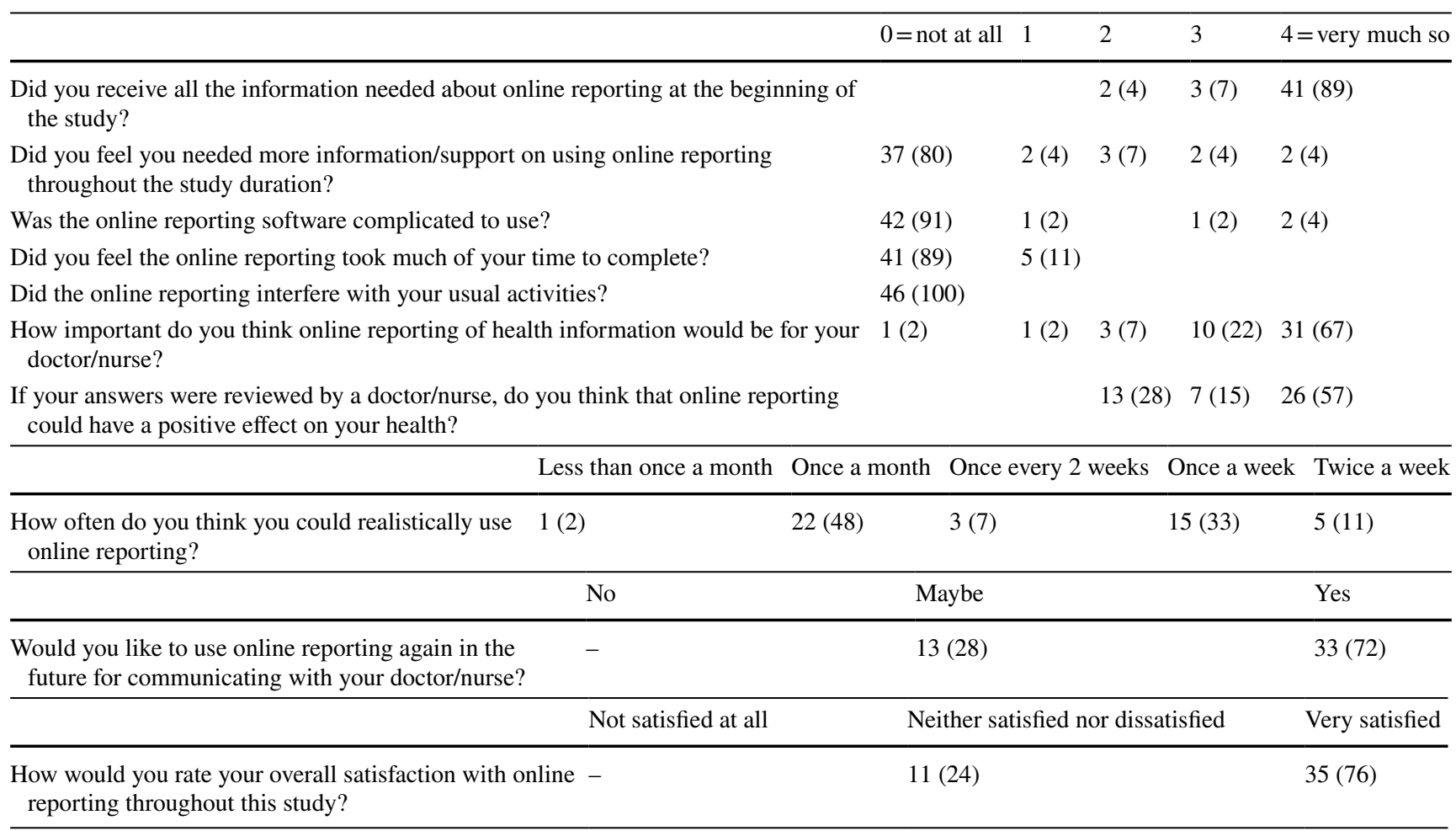

All data are shown as n (\%) unless otherwise indicated 


\section{Discussion}

This pilot study was conducted to assess the overall feasibility and acceptability of online PRO2-based remote reporting of disease activity in UC. We focused on golimumab-treated UC patients as there are limited real-world disease activity data available for this patient cohort. The patients in this study were asked to provide PRO2 scores online once every 4 weeks for a period of 6 months.

PRO2 scores were deemed a suitable target for remote reporting as this measure is brief and non-invasive, patients can self-report and it has been shown to correlate with more involved measures of disease severity, such as the full Mayo index [15]. The results of this pilot study suggest the patients studied were willing to participate across the duration of the study in the majority of cases and were able to use their own device to provide the requested data. Eighty-nine percent of patients completed every 4-weekly questionnaire, demonstrating that remote online reporting is feasible and long-term may provide patients with a more convenient alternative for disease management. Further research could be carried out with randomisation to treatment approach (remote monitoring vs traditional care) to assess remote monitoring over an extended duration with the aim of observing and comparing longer term outcomes.

Patients reported high satisfaction and acceptance of remote monitoring in addition to a high proportion of the sample stating that they would consider remote data reporting in future. However, fewer patients felt that this would have a positive impact on their health. Of note, the patient's physician/healthcare team did not have access to the patient PRO2 data during the study and were thus unable to make any therapeutic adjustments based on these scores. Accordingly, patients who experienced worsening of symptoms would not have seen any alterations to their care (e.g. earlier intervention) and so may have struggled to see the potential benefit from the remote reporting requirements. We recognise the opportunity for physician intervention based on disease activity reports as an important extension for future research. Despite this, patients appear optimistic about the use of remote reporting in the future.

Table 5 Healthcare resource utilisation at baseline (defined as 6 months preceding study enrolment) and 6-month study endpoint

\begin{tabular}{lll}
\hline & Baseline & 6 Months \\
\hline Routine consultations & $n=52$ & $n=51$ \\
Mean (SD), range & $2.1(1.51), 0-6$ & $1.7(1.86), 0-10$ \\
Telephone clinics & $n=52$ & $n=52$ \\
Mean (SD), range & $0.8(1.23), 0-5$ & $0.3(0.56), 0-2$ \\
Hospitalisations outside of & $n=52$ & $n=52$ \\
routine consultations & & \\
Mean (SD), range & $<0.1(0.19), 0-1$ & $0.2(0.69), 0-4$ \\
\hline
\end{tabular}

Data assessing current healthcare resource use indicated that on average, patients had face-to-face consultations with their healthcare team approximately twice every 6 months. Healthcare resource utilisation in the form of consultations remained consistently low from baseline to study end; a reflection on current clinical practice in this sample of UC patients. However, the addition of remote reporting to patient care could provide an opportunity for greater disease control, as well as reassurance to both the patient and treating site, with minimal impact to the healthcare system. Due to the nature of online reporting, if fully implemented, reported PRO2 scores could be made visible to the healthcare team immediately, enabling them to intervene if reported scores reach a certain level. Indeed, patients have previously expressed frustration with the traditional follow-up schedule for consultations and a preference to be seen when unwell [12]. The remote reporting schedule described here could offer a potential way of introducing this approach for some patients and may result in improved overall care for those involved, although this would need to be formally evaluated. However, of note, research suggests that clinical activity is not necessarily indicative of histological activity and patients free of clinical symptoms may still be experiencing mucosal inflammation [16]. In view of this, collection of PRO2 scores should be seen as additional information that could be used to inform care, but it should be kept in mind that patient reports of symptoms will not tell the whole story.

Due to the pilot nature of the study, the sample was based on a small proportion of patients who were initially willing to participate in a study of this nature. It is possible that this group is not representative of the UC patient population receiving golimumab. No formal data are available describing the number of patients who were not willing to participate and therefore, a potential sample bias must be considered when interpreting these results. We acknowledge that remote monitoring, if rolled out more formally, is not necessarily something that all patients will view favourably. However, it may become a potential offering that is appropriate for some patients, based on their personal preferences and disease status.

The increasing prevalence of inflammatory bowel diseases (IBD) globally requires population health management strategies to increase quality of care and patient outcomes, whilst reducing associated healthcare costs. In order to reduce the burden on the healthcare system, effective strategies are required to monitor patients and promptly manage flares. The implementation of remote systems to the patient care pathway is one strategy that could help in the development of a more efficient approach to IBD management. Atreja et al. observed the impact of remote monitoring on quality of care and quality of life in IBD patients [17]. The results from the trial suggest that the incorporation of telemedicine technology can support the tracking of IBD patients and enhancing quality of life and quality of care. 


\section{Conclusion}

The results from this study support the feasibility of remote monitoring systems in UC using PRO2 as a measure of disease activity. Over the long term, this could provide patients with a convenient option for improved disease management.

Acknowledgements The authors would like to thank Derek Ho for preparing the draft manuscript on behalf of Adelphi Real World in accordance with the European Medical Writers Association guidelines. All authors are fully responsible for all content and editorial decisions for this manuscript.

Data Availability Statement The datasets generated during the current study are not publicly available due to concerns around patient privacy, but a limited data set is available from the corresponding author on reasonable request.

\section{Compliance with Ethical Standards}

This study is supported by Merck \& Co., Inc, New Jersey. The authors are fully responsible for all content and editorial decisions for this manuscript. SS received honoraria from Merck \& Co., Inc for a consultancy role in this work. JR, JW and DJ are employees of Adelphi Real World. CB and RD are employees of Merck Sharp \& Dohme Ltd., Hoddesdon, UK. SK is an employee of Merck \& Co., Inc., Kenilworth, NJ, USA.

Open Access This article is distributed under the terms of the Creative Commons Attribution-NonCommercial 4.0 International License (http://creativecommons.org/licenses/by-nc/4.0/), which permits any noncommercial use, distribution, and reproduction in any medium, provided you give appropriate credit to the original author(s) and the source, provide a link to the Creative Commons license, and indicate if changes were made.

\section{Appendix}

Please indicate how you perceive your stool frequency (Based on the last 3 days)

$\square$ Normal

$\square$ 1-2 more stools than normal

$\square$ 3-4 more stools than normal

$\square 5+$ more stools than normal

Please indicate the severity of your rectal bleeding (Based on the last 3 days)

$\square$ No blood seen

$\square$ Streaks of blood seen with stools for half of the time

$\square$ Obvious blood with stool most of the time

$\square$ Blood alone passed (with no stool)

\section{References}

1. da Silva BC, Lyra AC, Rocha R, Santana GO. Epidemiology, demographic characteristics and prognostic predictors of ulcerative colitis. World J Gastroenterol. 2014;20(28):9458-67.
2. Ho EY, Cominelli F, Katz J. Ulcerative colitis: what is the optimal treatment goal and how do we achieve it? Curr Treat Options Gastroenterol. 2015;13(1):130-42.

3. Sandborn WJ, et al. Subcutaneous golimumab maintains clinical response in patients with moderate-to-severe ulcerative colitis. Gastroenterology. 2014;146:96-109.e1.

4. Loftus EV. Emerging diagnostic methods in inflammatory bowel disease. Gastroenterol Hepatol (N Y). 2007;3(4):284-6.

5. Dranga M, Mihai C, Drug V, Dumitrescu G, Prelipcean CC. A rapid test for assessing disease activity in ulcerative colitis. Turk J Gastroenterol. 2016;27(2):149-55.

6. Garud S, Peppercorn MA. Ulcerative colitis: current treatment strategies and future prospects. Therap Adv Gastroenterol. 2009;2(2):99-108.

7. Bewtra M, Brensinger CM, Tomov VT, Hoang TB, Sokach CE, et al. An optimized patient-reported ulcerative colitis disease activity measure derived from the Mayo score and the simple clinical colitis activity index. Inflamm Bowel Dis. 2014;20(6):1070-8.

8. Jairath V, Khanna R, Zou GY, Stitt L, Mosli M, et al. Development of interim patient-reported outcome measures for the assessment of ulcerative colitis disease activity in clinical trials. Aliment Pharmacol Ther. 2015;42(10):1200-10.

9. Lewis JD, Chuai S, Nessel L, Lichtenstein GR, Aberra FN, et al. Use of the noninvasive components of the Mayo score to assess clinical response in ulcerative colitis. Inflamm Bowel Dis. 2008;14(12):1660-6.

10. Marin-Jimenez I, Nos P, Domenech E, Riestra S, Gisbert JP, et al. Diagnostic performance of the simple clinical colitis activity index self-administered online at home by patients with ulcerative colitis: CRONICA-UC study. Am J Gastroenterol. 2016;111(2):261-8.

11. Commission E. Communication from the commission to the European Parliament, The Council, The European Economic And Social Committee And The COMMITTEE OF THE REGIONS 2012. https://ec.europa.eu/health//sites/health/files/ehealth/docs/ com_2012_736_en.pdf.

12. Aguas Peris M, Del Hoyo J, Bebia P, Faubel R, Barrios A, et al. Telemedicine in inflammatory bowel disease: opportunities and approaches. Inflamm Bowel Dis. 2015;21(2):392-9.

13. Kemp K, Griffiths J, Campbell S, Lovell K. An exploration of the follow-up up needs of patients with inflammatory bowel disease. J Crohns Colitis. 2013;7(9):e386-95.

14. de Jong MJ, Huibregtse R, Masclee AM, Jonkers DS, Pierik MJ. Patient-reported outcome measures for use in clinical trials and clinical practice in inflammatory bowel diseases: a systematic review. Clin Gastroenterol Hepatol. 2018;16:648-63.

15. Dhanda AD, Creed TJ, Greenwood R, Sands BE, Probert CS. Can endoscopy be avoided in the assessment of ulcerative colitis in clinical trials? Inflamm Bowel Dis. 2012;18:2056-62.

16. Baars JE, Nuij VJAA, Oldenburg B, Kuipers EJ, van der Woude CJ. Majority of patients with inflammatory bowel disease in clinical remission have mucosal inflammation. Inflamm Bowel Dis. 2012;18:1634-40.

17. Atreja A, Khan S, Otobo E, Rogers J, Ullman T, Grinspan A, Itzkowitz S, Maser E, Cohen B, Colombel J-F, Sands B. P554 Impact of real world home based remote monitoring on quality of care and quality of life in inflammatory bowel disease patients: one year results of pragmatic randomized trial. https://www.eccoibd.eu/publications/congress-abstract-s/abstracts-2017/item/p554impact-of-real-world-home-based-remote-monitoring-on-quali ty-of-care-and-quality-of-life-in-inflammatory-bowel-diseasepatients-one-year-results-of-pragmatic-randomized-trial-2.html. Accessed 31 May 2018. 